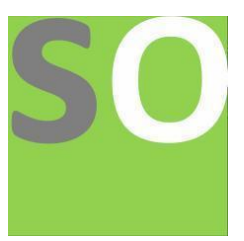

Article title: A Novel Computer Algorithm for Automating Nano-Scale Thickness Identification of 2D Materials Authors: Enoch Xu[1]

Affiliations: University of California Riverside[1]

Orcid ids: 0000-0001-9999-3134[1]

Contact e-mail: enoch.t.xu@gmail.com

License information: This work has been published open access under Creative Commons Attribution License http://creativecommons.org/licenses/by/4.0/, which permits unrestricted use, distribution, and reproduction in any medium, provided the original work is properly cited. Conditions, terms of use and publishing policy can be found at https://www.scienceopen.com/.

Preprint statement: This article is a preprint and has not been peer-reviewed, under consideration and submitted to ScienceOpen Preprints for open peer review.

DOI: 10.14293/S2199-1006.1.SOR-.PPUG1GE.v1

Preprint first posted online: 27 January 2021

Keywords: graphene, computer vision, 2D materials, Raman spectroscopy, 2D material thickness, optical characterization 


\title{
A Novel Computer Algorithm for Automating Nano-Scale Thickness Identification of 2D Materials
}

\author{
Enoch Xu
}




\section{Abstract}

Atomically thin 2D materials such as graphene promise great innovation in the electronics industry. However, these promises have stayed unfulfilled due to the lack of commercial 2D material production. There is currently no scalable and no economical way to identify the thicknesses of 2D materials reliably. Traditionally, Raman spectroscopy or atomic force microscopy (AFM) is used to measure the nanoscale (1-10 atom) thicknesses of 2D materials. These processes have many limitations because they require expensive equipment, are highly time-consuming, and only provide data for a single point on the sample. This paper presents a cheap and scalable solution that uses optical microscopy images to identify thicknesses. Two significant challenges need to be overcome to make this method feasible. Firstly, color temperatures and lighting intensities vary between different cameras and microscope setups. Secondly, the atomic-scale thicknesses of 2D materials translate into a very low contrast ratio between the 2D material and the substrate. This paper addresses these two challenges with novel computer vision and color normalization techniques. The method's accuracy was verified by comparing its results with measurements from Raman spectroscopy. It reliably calculates thicknesses with a single compressed jpg image, making it drastically faster and cheaper than traditional methods. The algorithm can be applied to any 2D material stack combination and adapt to any microscope setup, regardless of camera type and light color temperature. Furthermore, while the Raman spectrometer uses a laser to measure a single point's thickness, the new method provides insight into the topography of the entire material surface. All these advancements pave the way for industrial-scale production of 2D materials. 


\section{Introduction}

Imagine a future where your phone lasts a whole week on a single 10-minute charge, and your tablet rolls up like a newspaper. Recently, researchers have proved that these technologies are possible with atomically thin 2D materials. For example, researchers at Samsung have recently developed such a battery using graphene. Graphene has the highest conductivity of any known material. However, due to the nanoscale-thicknesses of 2D materials, even slight changes in these values can completely change their properties. For example, bilayer graphene is only $\sim 0.35 \mathrm{~nm}$ thicker than monolayer graphene, yet it is used for entirely different purposes since monolayer graphene's electron mobility is more than 10 times greater than that of bilayer graphene. Traditionally, the thickness (atomic layer count) of 2D materials is measured by Raman spectroscopy or atomic force microscopy (AFM). These methods only provide data on a small region while being laborious and time-consuming. Since there is no scalable thickness identification method, futuristic technologies made possible by $2 \mathrm{D}$ materials cannot come to the mass market.

This paper presents a cheaper, more efficient method using microscope images to identify thickness, accelerating 2D material research and opening possibilities for industrial production. The algorithm consists of three main parts. In Part I, the optics transfer-matrix method and colorimetry conversions are used to calculate RGB values for different 2D material stack combinations. In Part II, a separate computer vision process segments images to separate 2D samples from the substrate. Since traditional segmentation methods such as the Canny edge detector fail to work under the low contrast ratios presented by 2D materials, Part II develops a novel computer vision process to overcomes these issues. Part III brings the first two parts together to determine the thickness. It overcomes the challenge of inconsistent lighting and camera settings through a novel color normalization technique.

The accuracy of the algorithm was verified through a comparison process with the traditional Raman method. 2D material samples were micro-mechanically exfoliated in the lab using the Nobel Prize-winning "Scotch tape method" [10]. The thickness is measured by both the algorithm and the Raman spectrometer to create an accuracy benchmark. 


\section{Part I - Color Derivation of the 2D Material Stack}

The color derivation algorithms calculate the sRGB' value given any defined stack of 2D materials by deriving a spectral curve from the transfer-matrix method for thin-films, then converting the spectral curve into the universal sRGB' color space. The calculated sRGB' values of 2D material stacks are later compared with sRGB' values from optical images to determine thickness. In addition, the substrate color is computed to provide a reference for color normalization in Part III.

\subsection{Transfer-Matrix Method for Multilayer Systems}

The transfer-matrix method is a mathematical model to analyze the propagation of electromagnetic waves in a multilayer structure, such as those in 2D materials or other thin films. The structure consists of a stack of material layers, each with a thickness $d_{i}$ and refractive indices $n_{i}$. Each layer is separated by interference planes. In this paper, the transfer-matrix method is used to produce the spectral curve ( $\lambda$ vs. reflectance) for stacks of 2D materials. The structure is regarded as an optical system with one input and one output.

Let $\mathrm{T}$ denote the transfer matrix of the multilayer stack. The complete transfer matrix can be calculated with the multiplication of all individual transfer matrices:

$$
T_{0 N}=T_{01} T_{1} T_{12} T_{2} \ldots T_{(N-1)} T_{(N-1) N}
$$

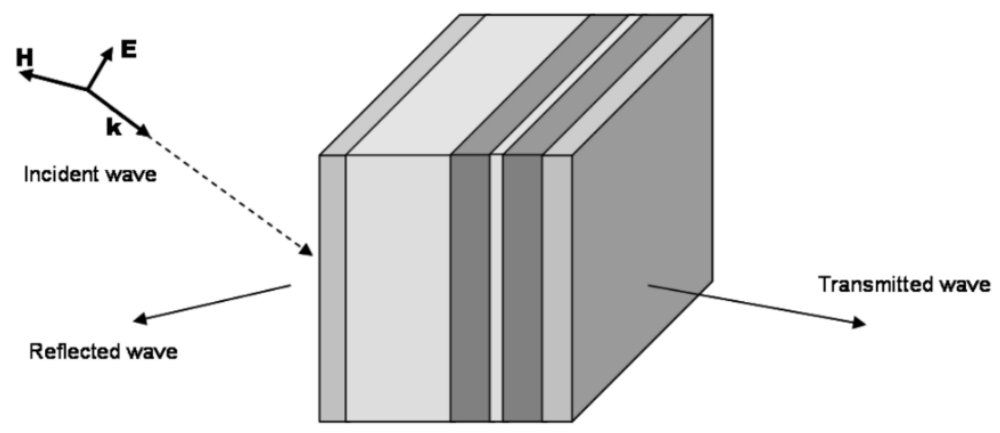

Fig. 1: Reflection and transmission at a multilayer stack. [1]

The transfer matrix $\mathrm{T}_{i j}$ for wave propagation through an interface between layers $i$ and $j$ can be calculated by: 


$$
\mathrm{T}_{i j}=\frac{1}{t_{i j}}\left[\begin{array}{cc}
1 & r_{i j} \\
r_{i j} & 1
\end{array}\right]
$$

where $r_{i j}$ and $t_{i j}$ are the Fresnel coefficients describing reflection and transmission between mediums for TM-polarization. They are given by:

$$
r_{i j}=\frac{n_{j} \cos \theta_{i}-n_{i} \cos \theta_{j}}{n_{j} \cos \theta_{i}+n_{i} \cos \theta_{j}}, \quad t_{i j}=\frac{2 n_{i} \cos \theta_{i}}{n_{j} \cos \theta_{j}+n_{i} \cos \theta_{j}}
$$

$n_{i}$ and $n_{j}$ are the refractive indices of the layers. $\theta_{i}$ and $\theta_{j}$ are the ray angles for layer $i$ and $j$, respectively. The refractive indices include real and imaginary components that change depending on the wavelength of light. A linear interpolation is applied to data from prior research experiments (ex: Fig. 2) [2][3][4].

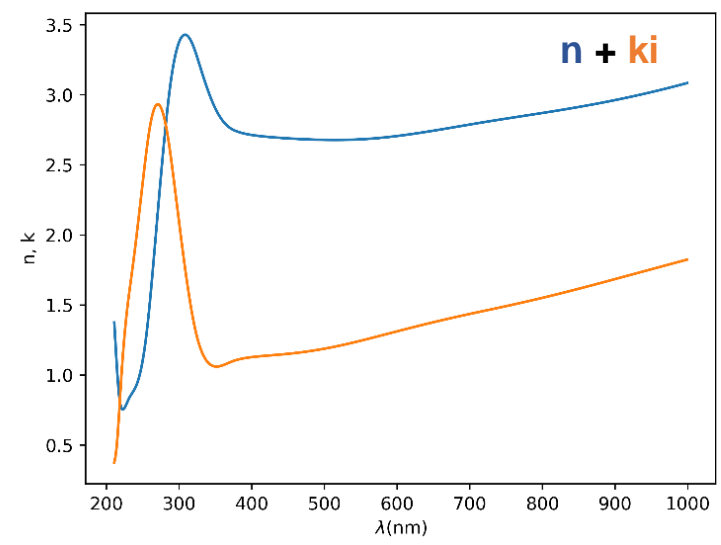

Fig. 2: The real and imaginary refractive indices $(n+k i)$ of graphene, which is entered into the equations to calculate the Fresnel coefficients.

The transfer matrix $\mathrm{T}_{i}$ for wave propagation through layer $i$ is given by:

$$
\mathrm{T}_{i}=\left[\begin{array}{cc}
e^{j \Phi_{i}} & 0 \\
0 & e^{-j \Phi_{i}}
\end{array}\right]
$$

where the phase change, $\Phi_{i}$ can be calculated by:

$$
\Phi_{i}=\frac{2 \pi}{\lambda_{0}} n_{i} d_{i} \cos \theta_{i}
$$

where $d_{i}$ is the thickness of layer $i$.

Finally, the reflectance $r$ is calculated with the final transfer matrix: 


$$
r=\frac{T_{21}^{0 N}}{T_{11}^{0 N}}
$$

The function of reflectance $r$ over 290-830nm light (approx. visible light range) creates a spectral curve (see Fig. 3 and Fig. 4) that determines the color of a material stack.

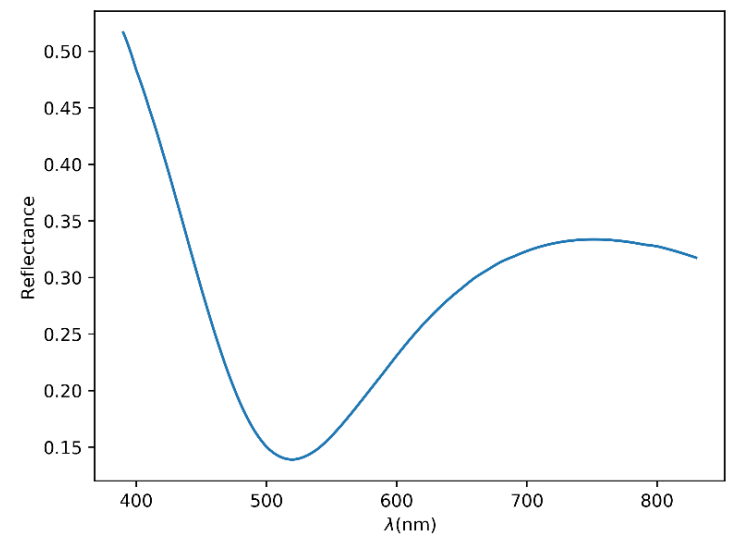

Fig. 3: The algorithm produces a spectral curve for wavelengths 290-830nm (approx. visible light range) for monolayer graphene ( $0.35 \mathrm{~nm}$ thick) on $285 \mathrm{~nm} \mathrm{SiO}$.

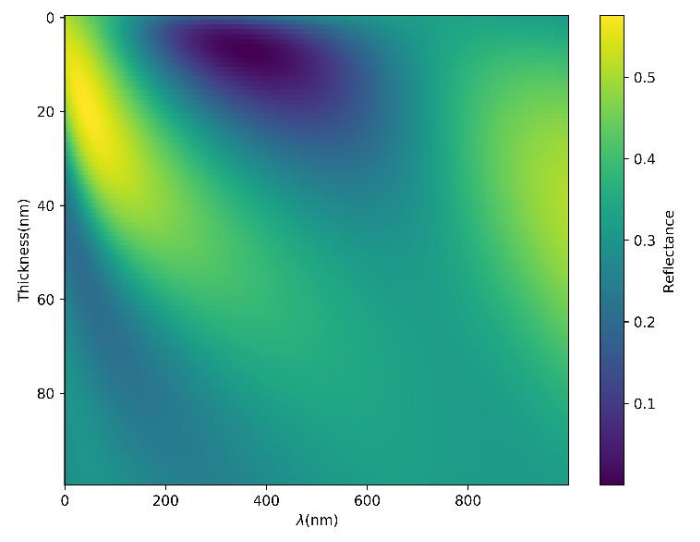

Fig. 4: The algorithm uses the transfer-matrix method to produce the graph above showing the reflectance of light from wavelengths $0-1000 \mathrm{~nm}$ for $0-100 \mathrm{~nm}$ graphite on $285 \mathrm{~nm} \mathrm{SiO}_{2}$. It is shown in the viridis color scale (Closer to yellow means higher value and closer to violet means lower value).

\subsection{CIE XYZ Colorspace}

To compare the spectral curves produced in section 2.1 with the colors captured in images, they must be translated into a digital representation. In this paper, it takes the form of a conversion to the universal CIE XYZ tristimulus values.

The CIE 1931 color spaces were created by the International Commission on Illumination (CIE) in 1931 to model the mathematical relationship between the wavelengths in the visible electromagnetic spectrum with human color vision. The standards are based on the experiments done by color scientists William David Wright and John Guild in the late 1920s [11]. The viewer would alter the brightness of 3 primary color beams $(436 \mathrm{~nm}, 546 \mathrm{~nm}, 700 \mathrm{~nm})$ until the test color was matched. The human eye uses three types of cone cells (SML), short S (420nm - 440nm), middle M (530nm - 540nm), and long L $(560 \mathrm{~nm}-580 \mathrm{~nm})$, each with distinct spectral sensitivity peaks. 
The CIE XYZ color space encompasses all color sensations of an average human being. Therefore, it is the standard device-invariant representation of color.

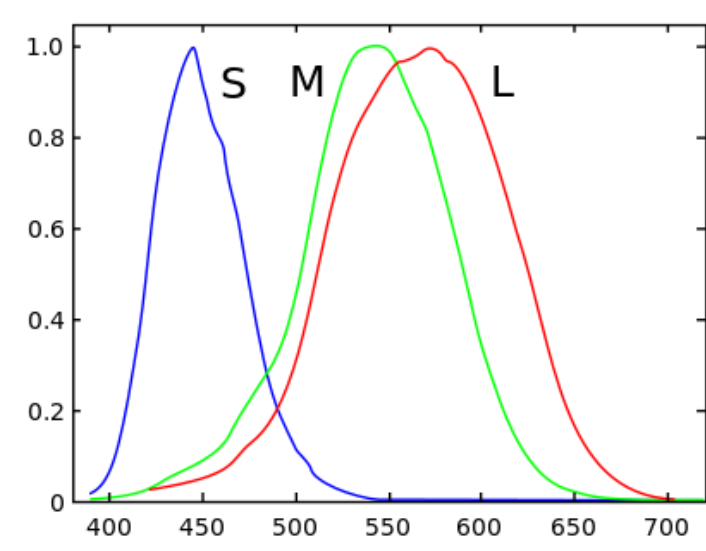

Fig. 5: The normalized spectral sensitivity of short- middle- and longhuman cone cells. [5]

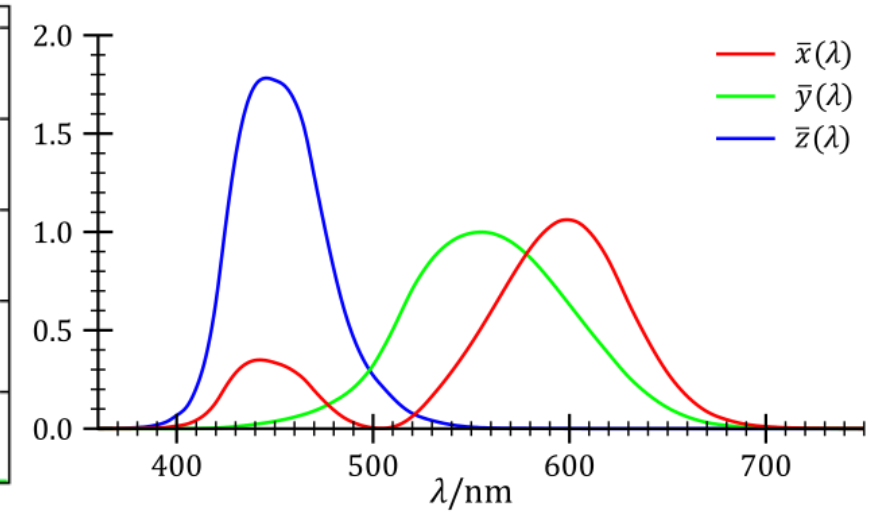

Fig. 6: The CIE XYZ standard observer color matching functions.

Tristimulus XYZ values are analogous to the human eye's LMS cone responses (see Fig. 5). Y is the luminance, $\mathrm{Z}$ is the s-cone response and is somewhat equal to blue, and the $\mathrm{X}$ value is a mix of cone response curves chosen to be non-negative for computational simplification.

Color-matching functions for XYZ (see Fig. 6) are the spectral sensitivity curves for X, Y, and Z. The color-matching functions (CIE 1931 standard curves) are fitted using the LevenbergMarquardt algorithm (non-linear least squares) [6]:

$$
\begin{gathered}
\bar{x}(\lambda)=1.065 e^{-\frac{1}{2}\left(\frac{\lambda-595.8}{33.33}\right)^{2}}+0.366 e^{-\frac{1}{2}\left(\frac{\lambda-446.8}{19.44}\right)^{2}} \\
\bar{y}(\lambda)=1.014 e^{-\frac{1}{2}\left(\frac{\ln \lambda-\ln 556.3}{0.075}\right)^{2}} \\
\bar{z}(\lambda)=1.839 e^{-\frac{1}{2}\left(\frac{\ln \lambda-\ln 449.8}{0.051}\right)^{2}}
\end{gathered}
$$

Based on the color-matching functions, the XYZ tristimulus values can be calculated by:

$$
X=\int_{390}^{830} S(\lambda) \bar{x}(\lambda) d \lambda, \quad Y=\int_{390}^{830} S(\lambda) \bar{y}(\lambda) d \lambda, \quad Z=\int_{390}^{830} S(\lambda) \bar{z}(\lambda) d \lambda
$$


where $S(\lambda)$ is the spectral power distribution and $\bar{x}(\lambda), \bar{y}(\lambda), \bar{z}(\lambda)$ are the CIE $1931 \mathrm{XYZ}$ colormatching functions. This mathematically derives the device-independent color values from the spectral curve of the stack.

\subsection{Device-Invariant XYZ to Device-Dependent sRGB'}

The device-independent XYZ Tristimulus values are converted into device-dependent sRGB' to match the camera's color space. The reverse conversion from D65 (roughly corresponding to the $6504 \mathrm{~K}$ color temperature of average midday light) XYZ values to sRGB' is done by multiplying by the inverse of conversion matrix $M[23]$ :

$$
\left[\begin{array}{l}
R_{\text {linear }} \\
G_{\text {linear }} \\
B_{\text {linear }}
\end{array}\right]=M^{-1} \cdot\left[\begin{array}{l}
X_{D 65} \\
Y_{D 65} \\
Z_{D 65}
\end{array}\right]
$$

where

$$
M=\left[\begin{array}{ccc}
0.412390799 & 0.357584339 & 0.180480788 \\
0.212639006 & 0.715168679 & 0.072192315 \\
0.019330819 & 0.11919478 & 0.950532152
\end{array}\right]
$$

Through this conversion process, a spectral curve, such as the one shown in Fig. 3, is given an sRGB' value based on the reflectance at each wavelength (see Fig. 6 and Fig. 7).

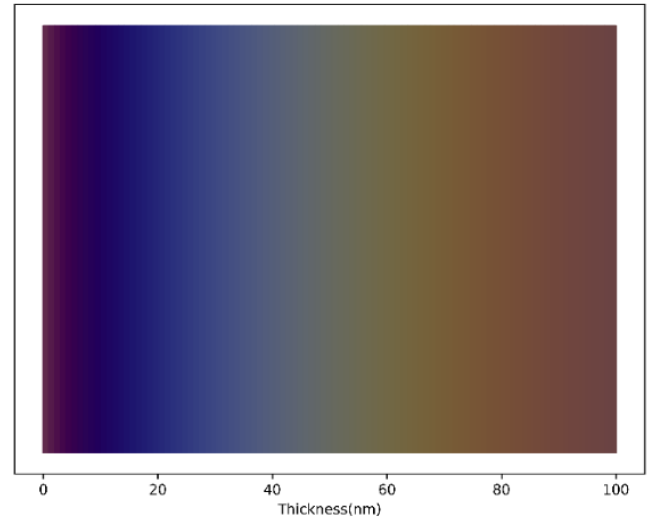

Fig. 6: The converted sRGB' values under D65 light for graphene thicknesses from $0-100 \mathrm{~nm}$.

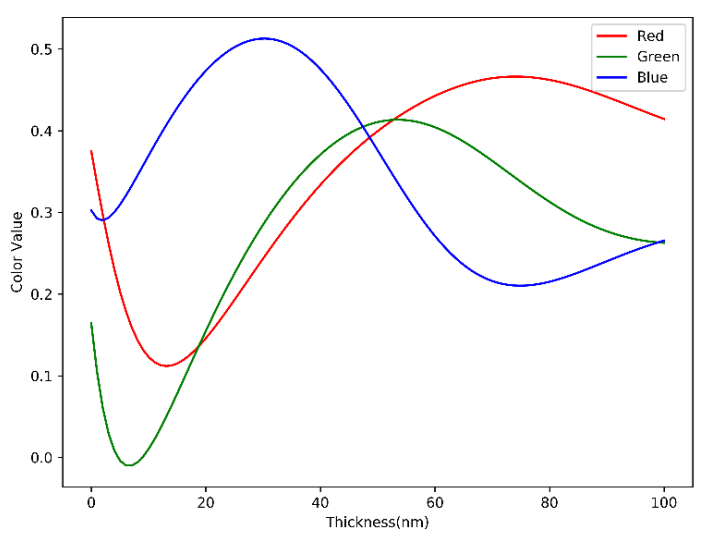

Fig. 7: The graph above shows how red, green, and blue values change for graphene on $\mathrm{SiO}_{2}$ substrate from thicknesses $0-100 \mathrm{~nm}$ 
Through Part I, the sRGB' value of any substrate or 2D material stack configuration is calculated.

\section{Part II - Image Segmentation}

For full automation of the thickness identification process, algorithms are applied to differentiate the 2D material sample from the substrate. This segmentation process is difficult because of the low contrast ratio between atom-thick 2D materials and the substrate. Furthermore, the images are compressed and noisy, so edge detectors such as the Canny method fail to work consistently. Instead, Part II develops a novel process employing Otsu's method.

\subsection{Gamma Expansion of Non-linear sRGB}

Before any other calculations or adjustments to the image, its color space should be linearized. Digital images are represented in the gamma-compressed (non-linear) sRGB color space. Humans perceive light differently than cameras do. When twice the number of photons hit the camera sensor, cameras receive twice the signal; however, it is only a fraction brighter to the human eye. The human eye is much more sensitive to changes in dark tones than changes in lighter tones since it allows vision to operate over a broader range of luminance. (Fig. 8)

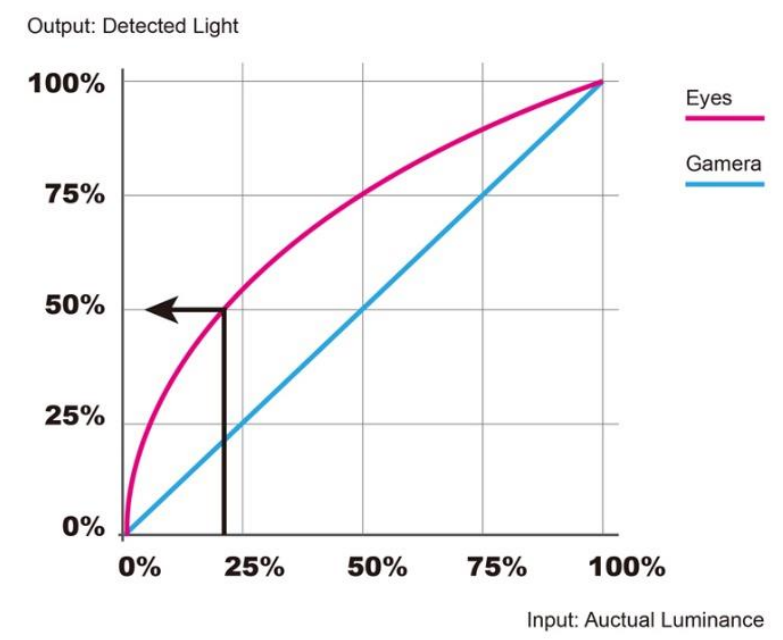

Fig. 8: Human Eye vs Camera Luminance Sensitivity [7]

By applying gamma correction, the usage of bits is optimized, and images match the non-linear perception of human eyes. (Fig. 9) 

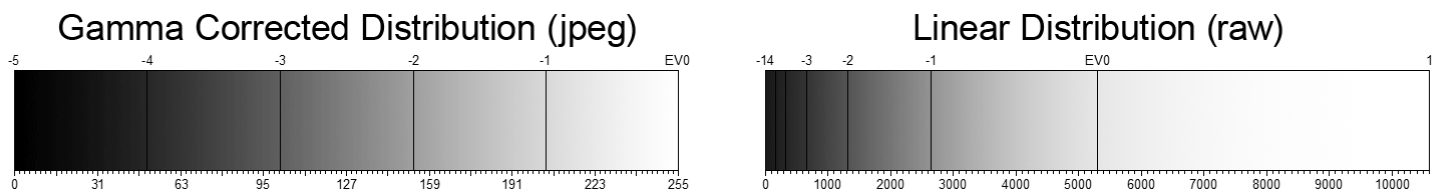

Fig. 9: Gamma corrected vs. linear encoding.

Linearizing sRGB values greatly simplifies computer vision processes such as color normalization; scalars and transformation matrices can be directly multiplied onto linear values. The non-linear sRGB values from the JPEG camera images are normalized to linear sRGB' using the following transformation:

$$
\gamma^{-1}(u)=\left\{\begin{aligned}
\frac{u}{12.92} & =\frac{25 u}{323} & & u \leq 0.0405 \\
\left(\frac{u+0.055}{1.055}\right)^{2.4} & =\left(\frac{200 u+11}{211}\right)^{\frac{12}{5}} & & \text { otherwise }
\end{aligned}\right.
$$

where $u$ is $R, G$, or $B$. (Fig. 10 shows linearization applied to an optical microscopy image of graphene used in this paper.)
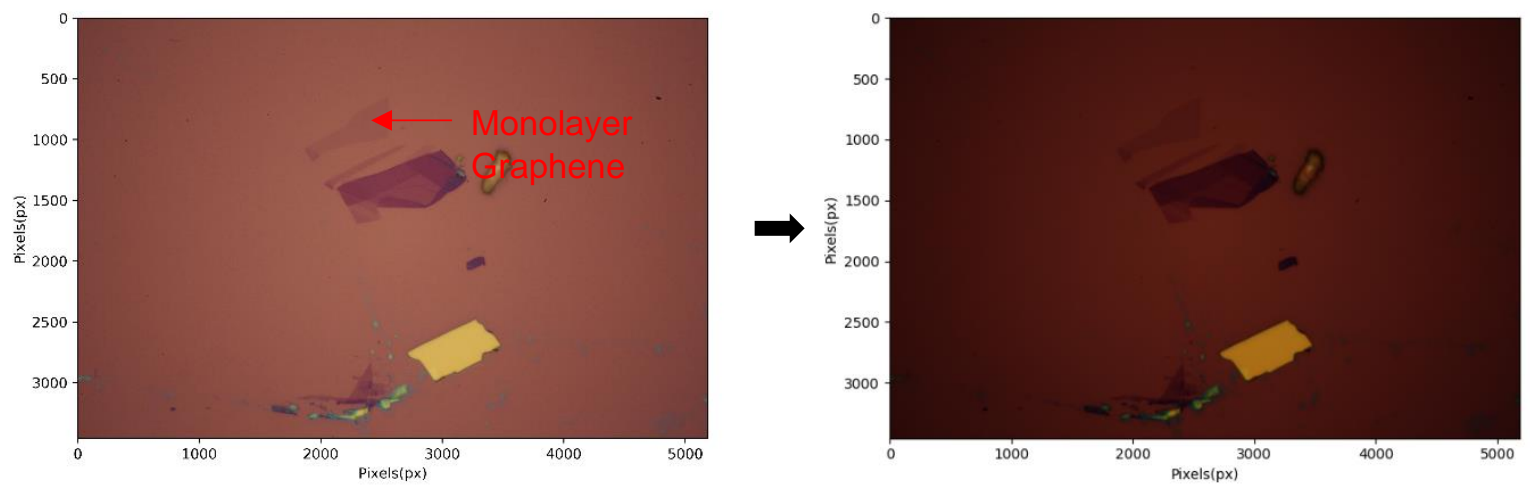

Fig. 10: Before (left) and after (right) the gamma expansion.

\subsection{De-vignetting}

After the linearization of the image, vignetting towards the corners is apparent. This is caused by the optics of microscope lenses and the radial light source on the microscope. It is critical to 
correct the non-uniformity in lighting intensities to simplify image segmentation and create an accurate means of color normalization.

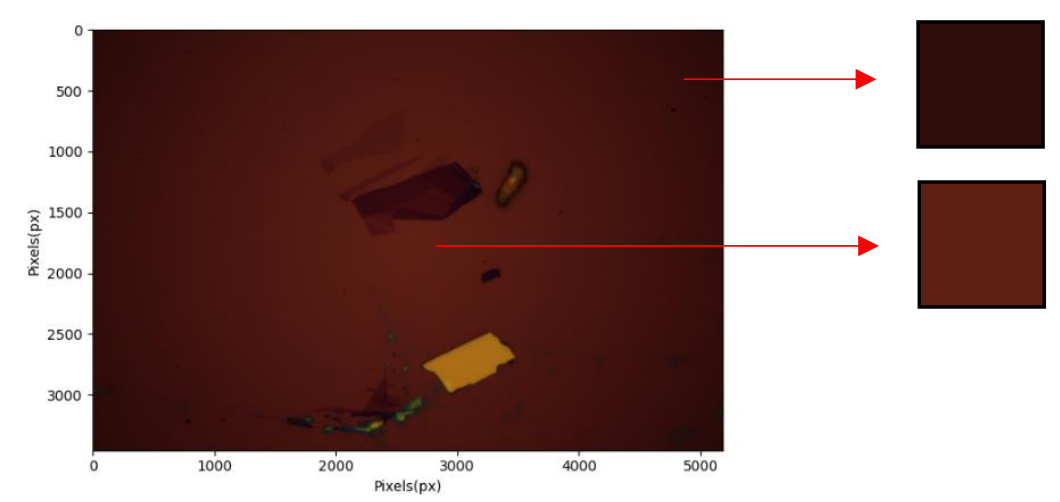

Fig. 11: Though the substrate is the same color throughout the image, Vignetting causes inaccuracies in intensity.

The lighting intensity map can be estimated as a 2D Gaussian function. Thus, generating a Gaussian kernel, then multiplying the pixel intensity values with the inverse of the kernel, can normalize the values.

The Gaussian kernel can be generated with:

$$
K(x, y)=\frac{1}{2 \pi \sigma^{2}} e^{\frac{-\left(x^{2}+y^{2}\right)}{2 \sigma^{2}}}
$$

where $x$ is the column of a pixel, $y$ is the row of a pixel, and $\sigma \approx \frac{x_{\text {final }}}{2}$.

The Gaussian kernel is then overlayed to produce the corrected image (Fig. 12):

$$
R_{\text {new }}(x, y)=\frac{R(x, y)}{K(x, y)}, \quad G_{n e w}(x, y)=\frac{G(x, y)}{K(x, y)}, \quad B_{n e w}(x, y)=\frac{B(x, y)}{K(x, y)}
$$



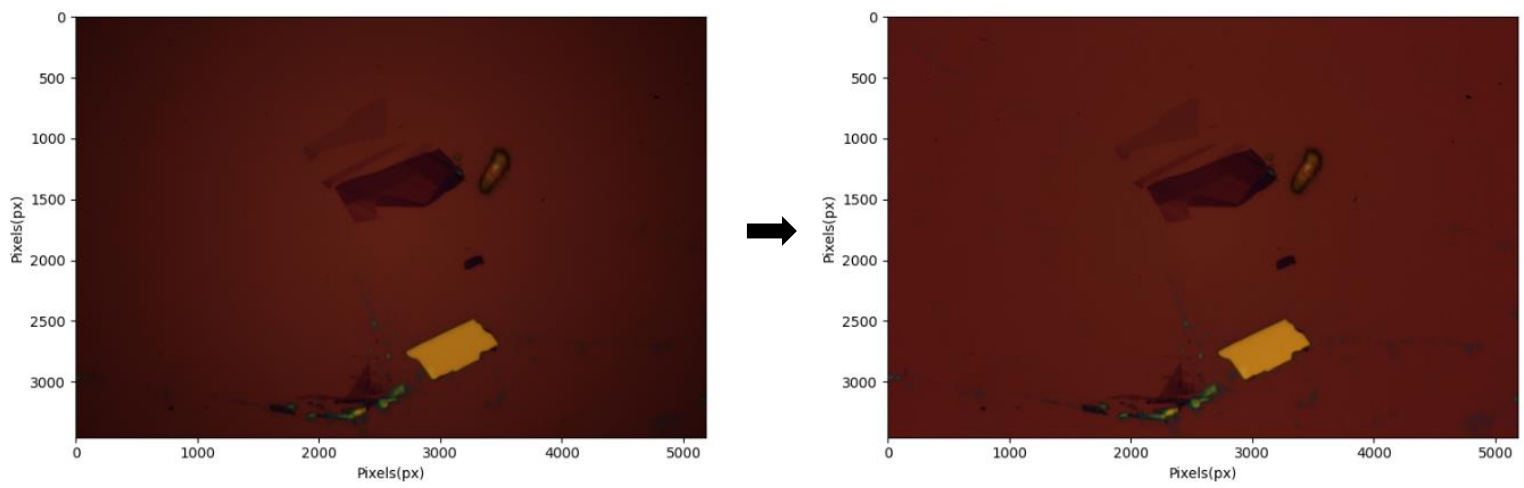

Fig. 12: The image becomes noticeably more uniform after the Gaussian kernel is applied.

\subsection{Conversion to Grayscale}

At this state, pre-processing is finished, so the image is ready for color normalization and computer vision calculations. Otsu's thresholding method is used to separate the 2D sample from the substrate. The method is binary, so it assigns each pixel a white or black value to separate the sample from the substrate. Thus, the three RGB color channels are converted into one luminance-preserving grayscale value to simplify the binarization process. This is given by:

$$
Y_{\text {linear }}=0.2126 R_{\text {linear }}+0.7152 G_{\text {linear }}+0.0722 B_{\text {linear }}
$$

where $Y_{\text {linear }}$ is the CIE 1931 linear luminance value, and the coefficients are determined based on the CIE standards for light intensity perception of human vision.
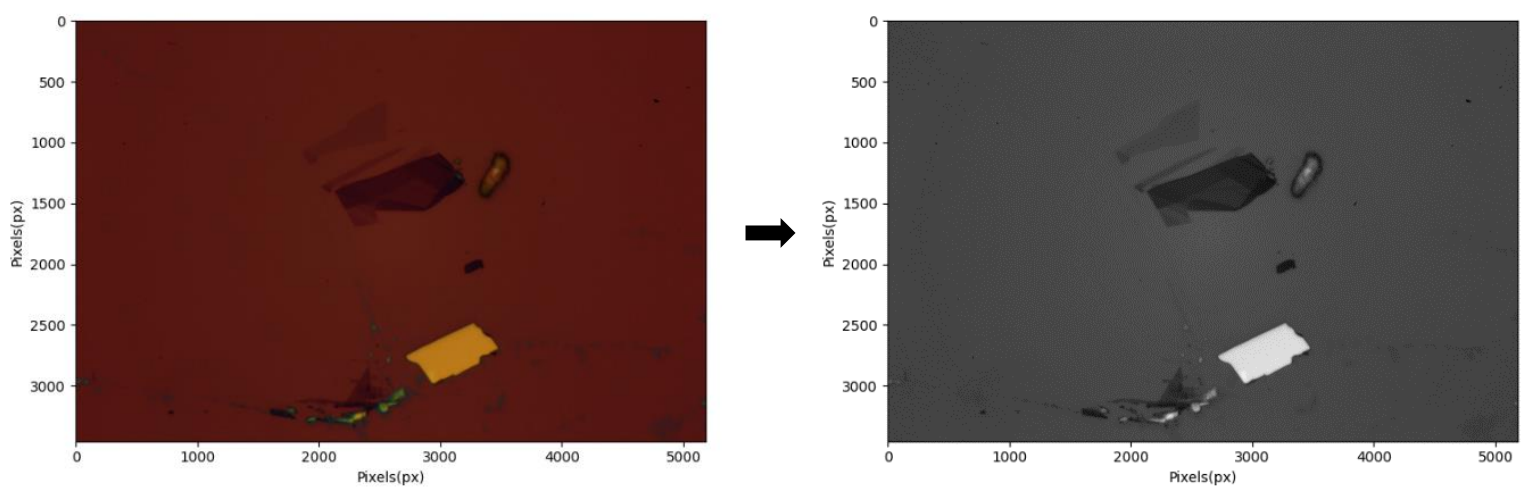

Fig. 13: The image before and after the grayscale conversion is applied. 


\subsection{Noise Reduction}

After converting the image to grayscale, any noise on the image needs to be cleaned up. Often, specks of noise can be perceived as pieces of material, causing errors in thresholding.
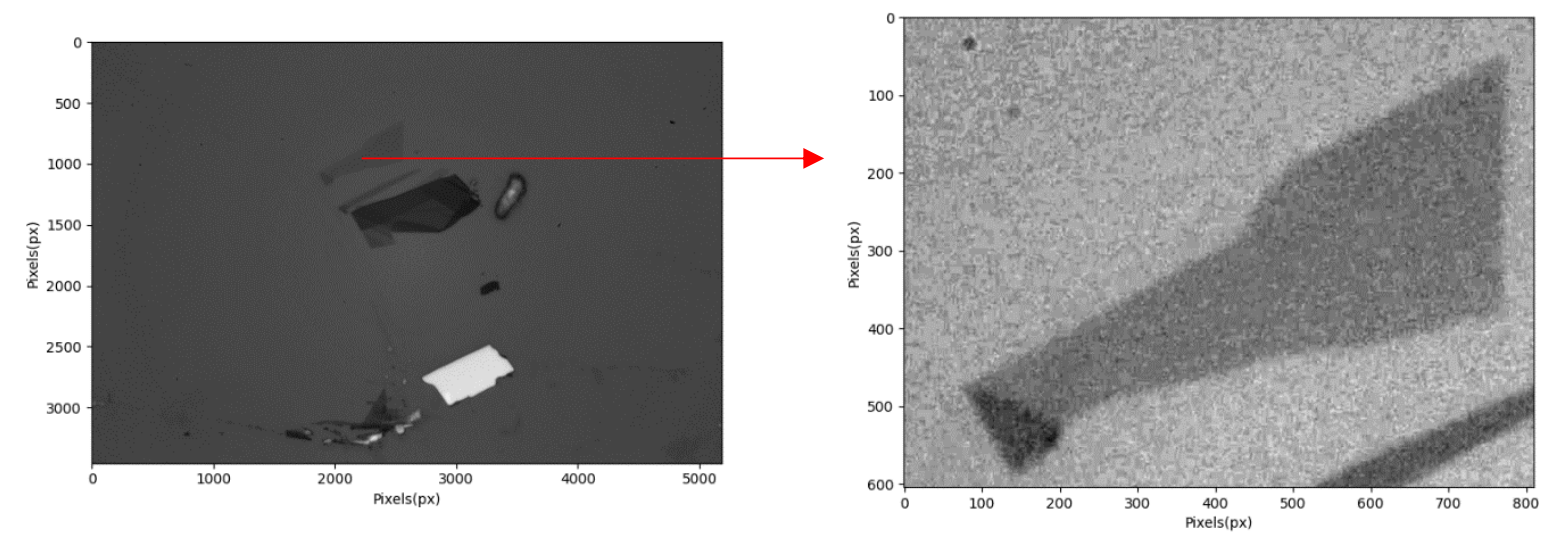

Fig. 14: A portion of the image is magnified to illustrate image noise.

The simplest way to remove image grain would be by averaging pixel values over a kernel (mean blur). However, a mean blur would soften edges, causing problems when thresholding samples. Instead, this paper employs a Gaussian blur so that each pixel is assigned the weighted average value of the pixels around it. Thus, noise is eliminated while preserving edges. The Gaussian kernel is created using a technique similar to the one used in de-vignetting (3.2), but the scale of the distribution is much smaller:

$$
K(x, y)=\frac{1}{2 \pi \sigma^{2}} e^{\frac{-\left(x^{2}+y^{2}\right)}{2 \sigma^{2}}}
$$

The Gaussian kernel produced is then applied via kernel convolution:

$$
G(x, y)=\sum_{d x=-a}^{a} \sum_{d y=-b}^{b} K(d x, d y) f(x+d x, y+d y)
$$

where $G(x, y)$ is the filtered image, and $f(x, y)$ is the original image, and $a$ and $b$ are used to traverse each column and row of the kernel, respectively. 

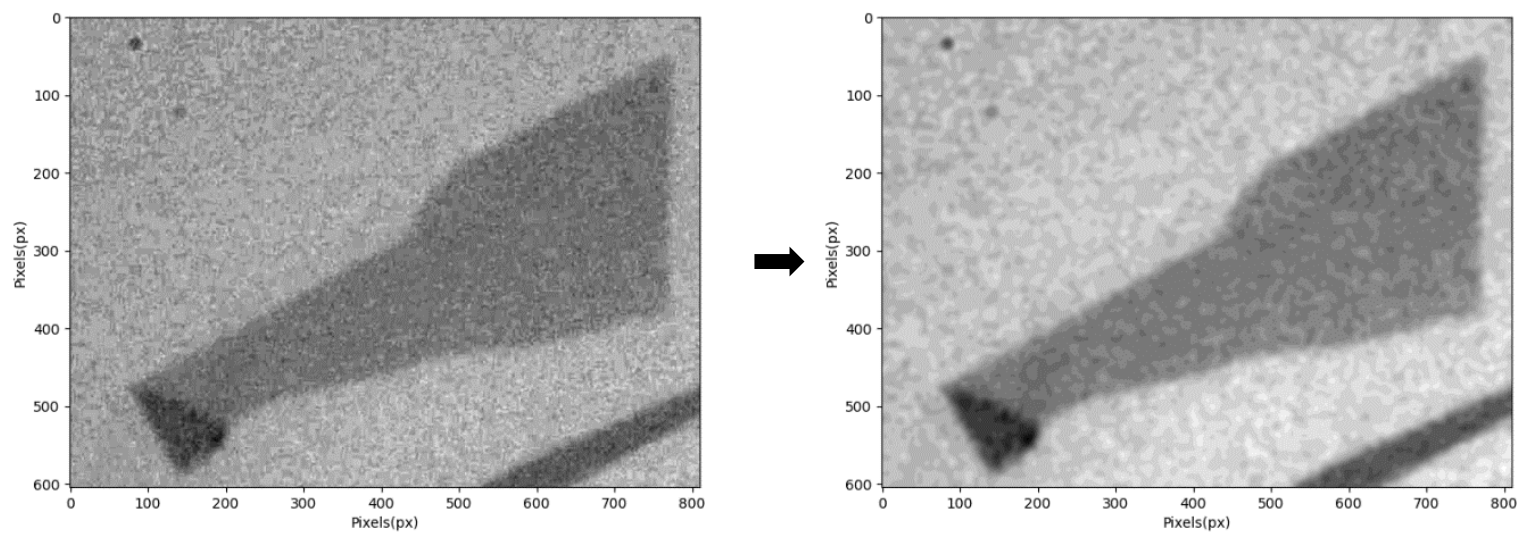

Fig. 15: Most noise is removed after the Gaussian kernel convolution is applied, making binarization much more accurate.

\subsection{Otsu's Method for Image Binarization}

Typically, arbitrary values are chosen to perform thresholding, but by applying Otsu's method [8], the threshold value $t$ can be determined automatically by minimizing the weighted intraclass variance in a bimodal image, which is defined by:

$$
\sigma_{w}^{2}(t)=\omega_{1}(t) \sigma_{1}^{2}(t)+\omega_{2}(t) \sigma_{2}^{2}(t)
$$

where the class probabilities $\omega_{1}(t)$ and $\omega_{2}(t)$ are:

$$
\omega_{1}(t)=\sum_{i=1}^{t} P(i), \quad \omega_{2}(t)=\sum_{i=t+1}^{L} P(i)
$$

and the variance $\sigma_{1}^{2}(t)$ and $\sigma_{2}^{2}(t)$ is:

$$
\sigma_{1}^{2}(t)=\frac{\sum_{i=1}^{t} i P(i)}{\omega_{1}(t)}, \quad \sigma_{2}^{2}(t)=\frac{\sum_{i=t+1}^{L} i P(i)}{\omega_{2}(t)}
$$

given that $L$ represent the bins of the image histogram (see Fig. 16). 


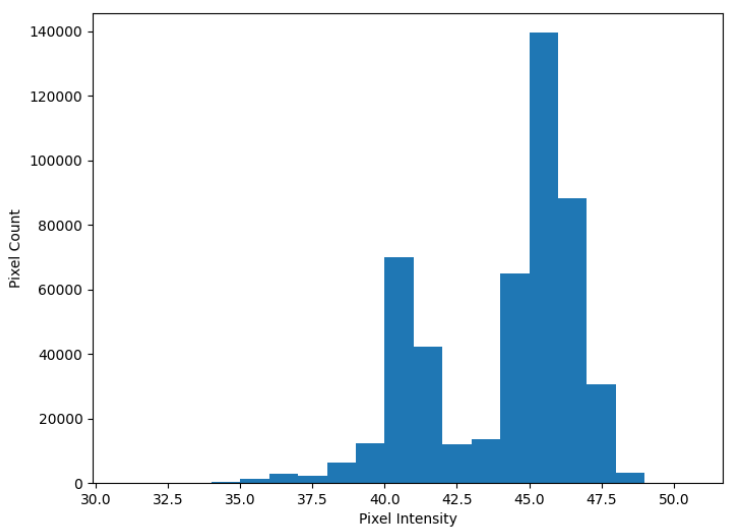

Fig. 16: The histogram of the image is created by plotting the number of pixels at each luminosity. There is a clear bimodal distribution.

The threshold $t$ can be found by iterating through each possible value to find the minimum. However, to increase the efficiency of the algorithm through a far faster recursive method, the minimizing of intra-class variance is computed by maximizing inter-class variance, which is given by:

$$
\sigma^{2}=\sigma_{w}^{2}(t)+\omega_{1}(t)\left[1-\omega_{1}(t)\right]\left[\mu_{1}(t)-\mu_{2}(t)\right]^{2}
$$

where the class means $\mu_{1}(t)$ and $\mu_{2}(t)$ are given by:

$$
\mu_{1}(t)=\sum_{i=1}^{t} \frac{i P(i)}{q_{1}(t)}, \quad \mu_{2}(t)=\sum_{i=t+1}^{L} \frac{i P(i)}{q_{2}(t)}
$$

After finding $t$, a thresholded version of the image is created by assigning each pixel a black or white value through a simple inequality:

$$
g(x, y)= \begin{cases}1, & I(x, y) \geq t \\ 0, & \text { otherwise }\end{cases}
$$


where $I(x, y)$ is the pixel intensity values between 0 and 255 .
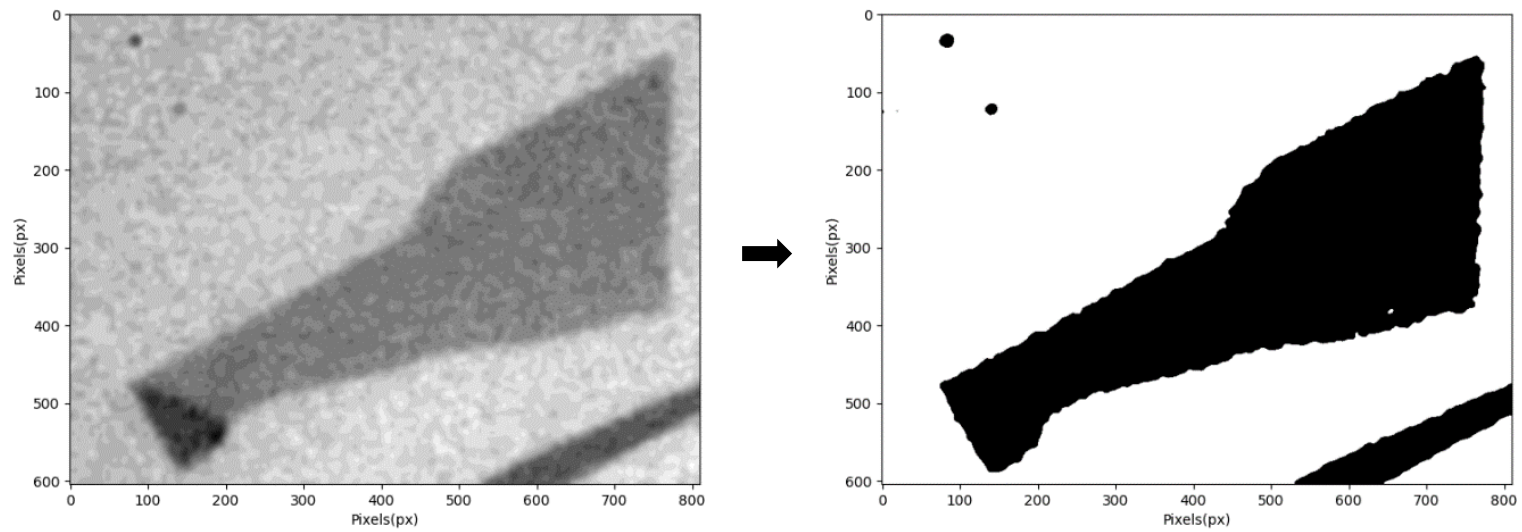

Fig. 17: Visualization of Otsu's binarization.

\subsection{Morphological Operations}

After applying Otsu's binarization, small traces of noise and holes may affect certain parts of the image. Thus, the morphological operation of opening is performed on the substrate.

Morphological opening consists of an erosion to fill in any white spots in the image, followed by dilation to remove noise and excess pixels created by erosion. This is performed with a simple structuring element $s$, which is a 9x9 ellipse. The erosion is given by:

$$
f \ominus s \equiv\left\{p \in \mathbb{Z}^{2} \mid(p+q) \in f, \text { for every } q \in s\right\}
$$
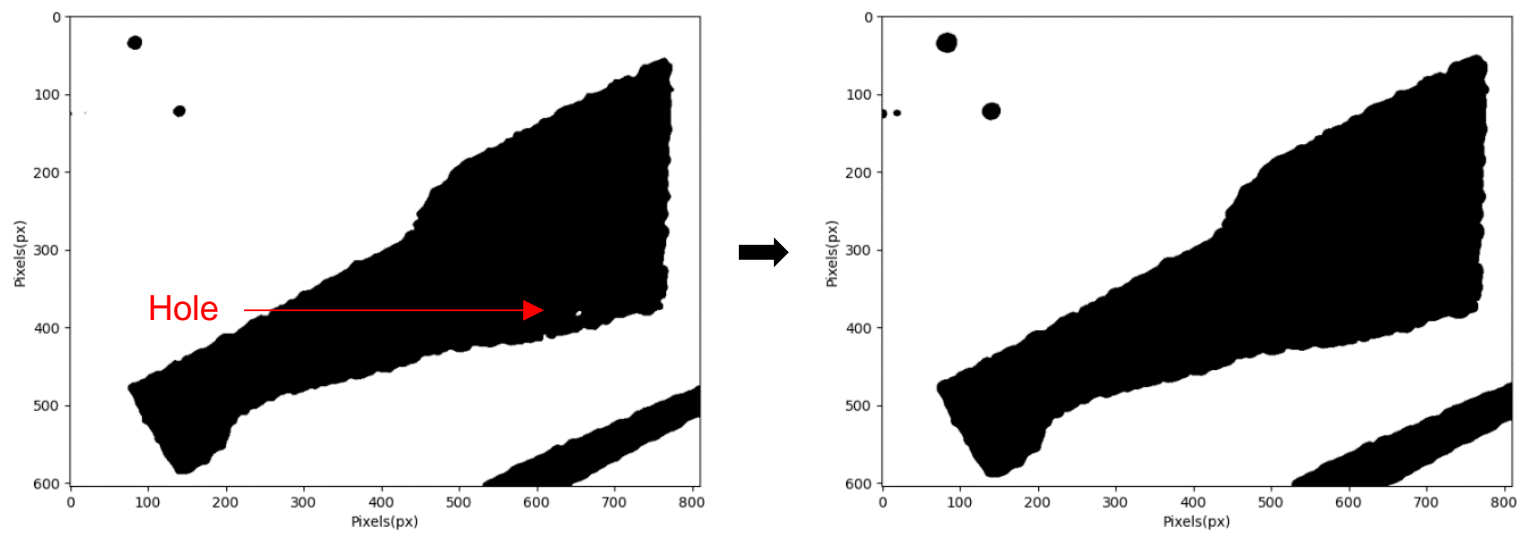

Fig. 18: After erosion(right), the holes in the sample are filled in, but the sample grows larger. 
and the dilation is given by:

$$
f \bigoplus s \equiv\{(p+q) \mid \text { for every } p \in F, q \in s\}
$$

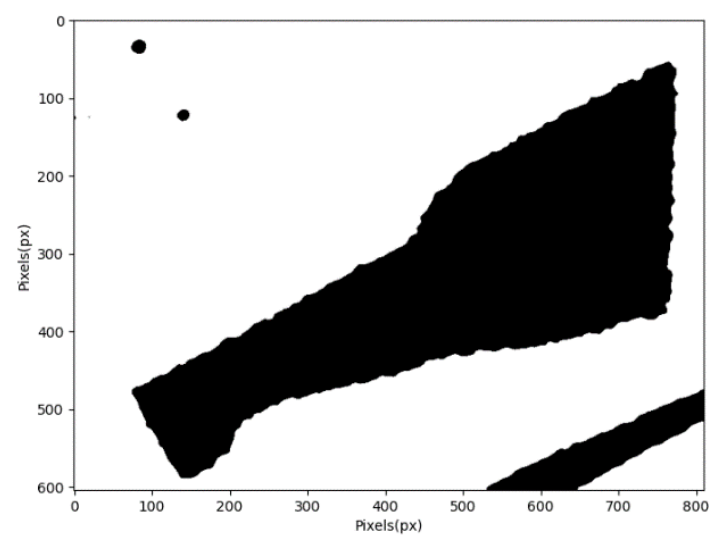

Fig. 19: After dilation, the sample returns to initial size while holes remain filled in, and noise is removed.

So, the new binary image resulting from opening is simplified to:

$$
g=f \circ s=(f \ominus s) \oplus s
$$

This intricate procedure creates a clean separation between the substrate and the sample, even with low-quality images.

\subsection{Contour Tracing}

This paper uses a method based on Suzuki's Contour Tracing Method [9] to segment and find RGB values needed for the color normalization technique. The tracing method efficiently draws the boundaries of the substrate and the sample.

Contours drawn from the binary image using Suzuki's method are applied onto a mask, which is overlayed to the original de-vignetted image from 3.2 to partition the image regions: 


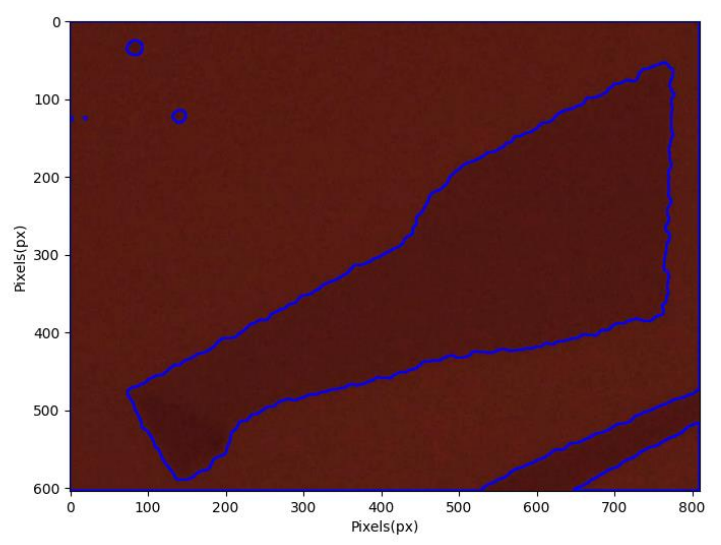

Fig. 20: The contours drawn are overlayed on the original de-vignetted image.

Now, the color of the substrate and the sample can be determined. This is done by sampling all the RGB values of pixels inside the contour mask and taking a simple mean. Averaging values from all the substrate pixels derives an accurate RGB value because errors that stem from sampling noisy pixels are eliminated.

\section{Part III - Color Normalization and Thickness Identification}

To identify thickness, colors calculated in Part I is compared with the sample's color in the image. However, the image's colors are inaccurate due to factors such as the white balance of the camera or the color temperature of the microscope's light. This paper develops a novel color normalization technique to correct the image and solve this problem.

\subsection{Color Normalization}

Since conditions such as bulb brightness and temperature, camera settings, and microscope setup change image colors, the image is normalized by applying the following transformation:

$$
\left[\begin{array}{l}
R \\
G \\
B
\end{array}\right]=M^{-1} \cdot\left[\begin{array}{l}
R^{\prime} \\
G^{\prime} \\
B^{\prime}
\end{array}\right], \quad M=\left[\begin{array}{ccc}
\alpha & 0 & 0 \\
0 & \beta & 0 \\
0 & 0 & \gamma
\end{array}\right]
$$

$R, G$, and $B$ are the color-corrected red, green, and blue components of the image; $R^{\prime}, G^{\prime}$, and $B^{\prime}$ are the pixel components before correction. Matrix $M$ models the influence of the illuminant 
color factors, so multiplying the inverse reverses the effects. The RGB illuminance coefficients $\alpha, \beta$, and $\gamma$ can be calculated using the substrate as a reference:

$$
\alpha=\frac{R_{\text {sub }}^{\prime}}{R_{\text {sub }}}, \quad \beta=\frac{G_{\text {sub }}^{\prime}}{G_{\text {sub }}}, \quad \gamma=\frac{B_{\text {sub }}^{\prime}}{B_{\text {sub }}}
$$

where $R_{\text {sub }}, G_{\text {sub }}$, and $B_{\text {sub }}$ are the color values for $285 \mathrm{~nm} \mathrm{SiO}_{2}$ derived in 2.3 , and $R_{\text {sub }}^{\prime}, G_{\text {sub }}^{\prime}$, and $B_{\text {sub }}^{\prime}$ are the average color values of the substrate in the given image.

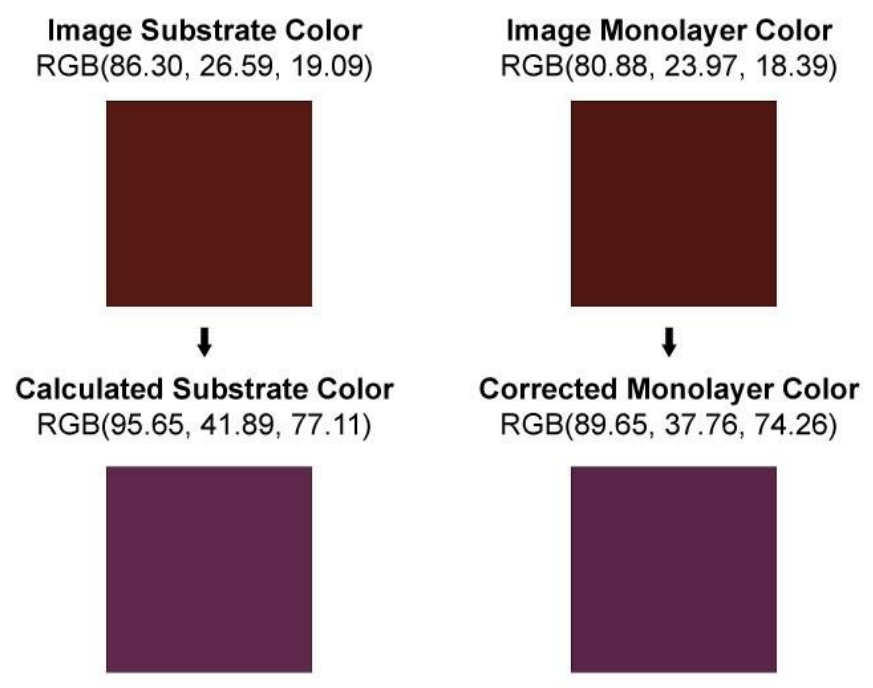

Fig. 21: The image normalization algorithm, visualized.
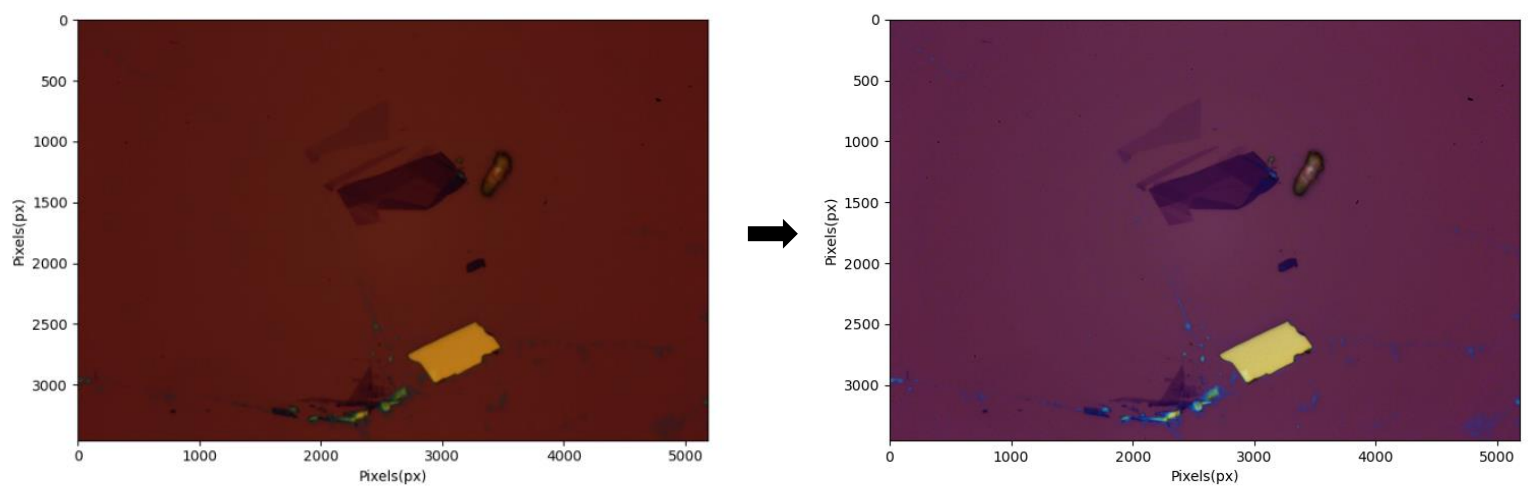

Fig. 22: Before(left) and after(right) image normalization. 


\subsection{Measuring Thickness}

2D material thickness is discrete since it is based on the atomic layer count. The thickness can be determined by minimizing the RGB difference between the normalized image RGB value (Fig. 23b) and the theoretical RGB values derived for each thickness in Part I (Fig. 23a). RGB difference is defined by:

$$
\Delta R G B=\sqrt{\left(R_{\text {calculated }}-R_{\text {norm }}\right)^{2}+\left(G_{\text {calculated }}-G_{\text {norm }}\right)^{2}+\left(B_{\text {calculated }}-B_{\text {norm }}\right)^{2}}
$$

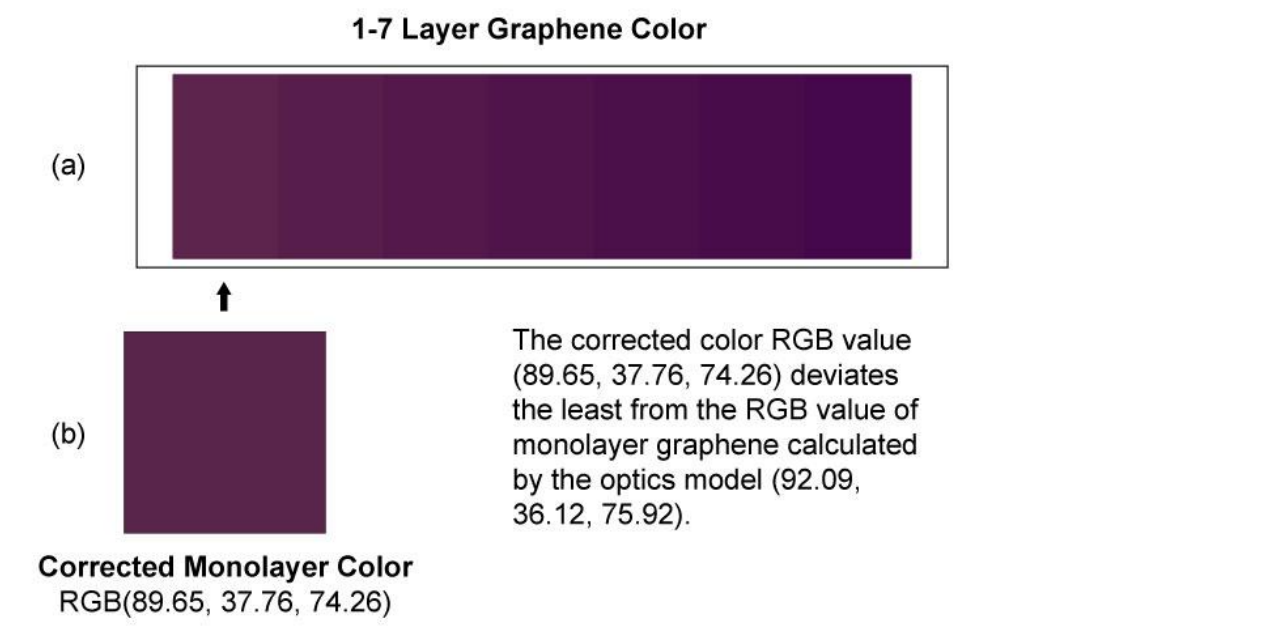

Fig. 23: The thickness identification process.

\section{Algorithm Accuracy Verification with Raman}

To verify the accuracy of the algorithm, results are compared with the traditionally used Raman method. Samples of 2D materials were micro-mechanically exfoliated onto silicon wafers with the "Scotch tape method." After finding suitable samples with an optical microscope, images were captured at varying illuminant parameters. The same samples were also scanned with a Raman spectrometer to compare the thickness measurement.

A sample's thickness can be determined with Raman spectroscopy by analyzing the ratio between the intensities of the 2D (around $2700 \mathrm{~cm}^{-1}$ ) and $\mathrm{G}$ peak (around $1580 \mathrm{~cm}^{-1}$ ) in the Raman spectra (Fig. 24). For example, in one of the samples tested, the 2D peak intensity was $\sim 150$, and the $\mathrm{G}$ peak had an intensity of $\sim 70$. This verified the sample to be monolayer graphene 
$(\sim 0.35 \mathrm{~nm}$ thick) since the ratio of the intensities for the $2 \mathrm{D}$ peak to the $\mathrm{G}$ peak was approximately two:

$$
\frac{I_{2 D}}{I_{G}} \approx 2
$$

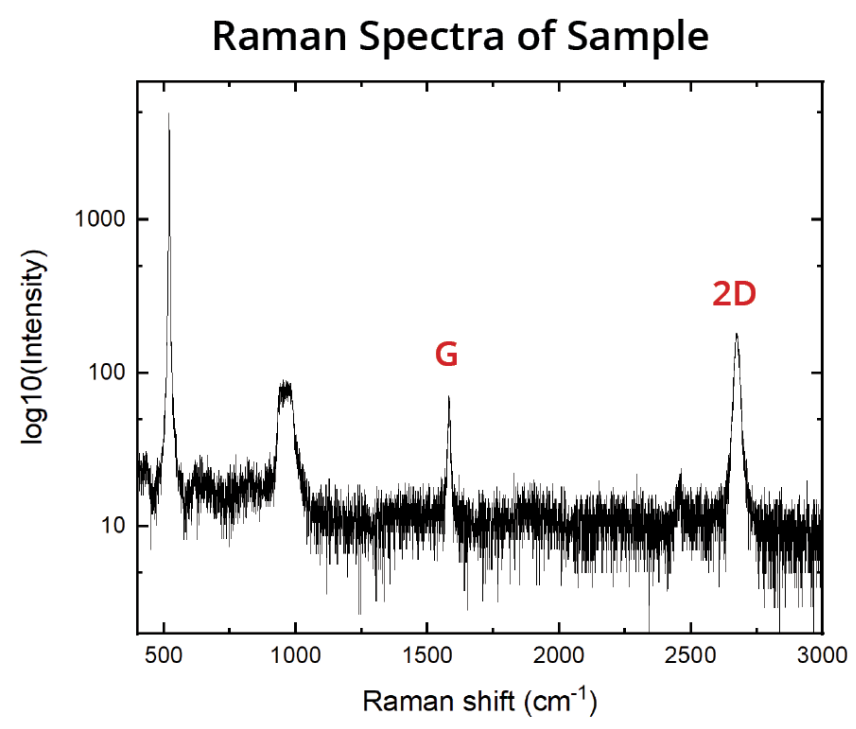

Fig. 24: The Raman Spectra of a monolayer graphene sample.

The algorithm's results were consistent with Raman spectroscopy for all samples tested. The algorithm developed matches the accuracy of Raman spectroscopy while producing results much faster and more cost-effectively. By only requiring compressed jpg optical images, the algorithm provides an automatable and scalable method that eliminates the need for expensive equipment.

\section{Conclusion}

The fully automated 2D material thickness identification algorithm developed in this paper is scalable to large quantities. Compared to previous attempts, this algorithm is much more flexible and extendable. The method requires no prior calibration or setup, so it can be rapidly implemented on any microscope setup and any 2D material stack combination. And by using a novel computer vision process, the algorithm can segment images despite the low contrast ratios between the 2D material and the substrate. Furthermore, the algorithm gives insight into the sample's entire surface instead of the single point measured by a Raman's laser. Combined with its speed and low cost, the algorithm opens possibilities for the mass production of 2D materials. 


\section{References}

[1] Figure 3.6. Reflection and transmission at a multilayer stack. Reprinted from "Transfer Matrix Formulation for Multilayer Systems," Photonics Research Group.

[2] Weber, J. W., et al. "Optical Constants of Graphene Measured by Spectroscopic Ellipsometry.” Applied Physics Letters, vol. 97, no. 9, 2010, p. 091904., doi:10.1063/1.3475393.

[3] Green, Martin A. "Self-Consistent Optical Parameters of Intrinsic Silicon at 300K Including Temperature Coefficients." Solar Energy Materials and Solar Cells, vol. 92, no. 11, 2008, pp. 1305-1310., doi:10.1016/j.solmat.2008.06.009.

[4] I. H. Malitson, "Interspecimen Comparison of the Refractive Index of Fused Silica*, $\dagger$," J. Opt. Soc. Am. 55, 1205-1209 (1965)

[5] Vanessaezekowitz at en.wikipedia, CC BY 3.0-2.5-2.0 1.0, via Wikimedia Commons

[6] C. Wyman, P. Sloan, P. Shirley, "Simple Analytic Approximations to the CIE XYZ Color Matching Functions," Journal of Computer Graphics Techniques, Vol. 2, No. 2, 2013.

[7] Accurate Gamma 2.2 And Pre-Set 5 Gamma Settings, color.viewsonic.com/explore/content/Accurate-Gamma_4.html.

[8] OTSU, NOBUYUKI. "A Tlreshold Selection Method from Gray-Level Histograms." Automatica 11 (1975): 285-296.

[9] Suzuki, Satoshi, and Keiichia Be. "Topological Structural Analysis of Digitized Binary Images by Border Following." Computer Vision, Graphics, and Image Processing, vol. 30, no. 1, 1985, pp. 32-46., doi:10.1016/0734-189x(85)90016-7.

[10] A. K. Geim and K. S. Novoselov, “The rise of graphene," Nature Materials, vol. 6, no. 3, pp. 183-191, 2007.

[11] Fairman, Hugh S., et al. "How the CIE 1931 Color-Matching Functions Were Derived from Wright-Guild Data." Color Research \& Application, vol. 22, no. 1, 1997, pp. 11-23., doi:10.1002/(sici)1520-6378(199702)22:1\&lt;11::aid-col4\&gt;3.0.co;2-7. 
[12] Tania Roy et al, "Field-Effect Transistors Built from All Two-Dimensional Material Components,” ACS Nano 8 (6), pp 6259-6264, 2014.

[13] J. Lei et al, "A facile method for precise layer number identification of two-dimensional materials through optical images," Optical Communications 440, February 2019.

[14] M. Born and E.Wolf," Principles of optics: electromagnetic theory of propagation, interference and diffraction of light," Oxford, Pergamon Press, 1964.

[15] G. Finlayson, B. Schiele, and J. Crowley and C. Ma, "Comprehensive Colour Image Normalization," Proc European Conference on Computer Vision, 1. 10.1007/BFb0055685, 1999.

[16] Abraham, Chandler. “A Beginner's Guide to (CIE) Colorimetry,” Medium, Color and Imaging, 15 Feb. 2019.

[17] Walker, John. “Colour Rendering of Spectra.” Colour Rendering of Spectra, 1996.

[18] Wall, Mark. "The Raman Spectroscopy of Graphene and the Determination of Layer Thickness," Thermo Fischer Scientific, 2011.

[19] Chen Y-F, Liu D, Wang Z-G, Li P-J, Hao X, Cheng K, Fu Y, Huang L-X, Liu X-Z, Zhang W-L and Li Y-R "Rapid Determination of the Thickness of Graphene Using the Ratio of Color Difference,” J. Phys. Chem. C 115 6690-3, 2011.

[20] Hai Li, Jumiati Wu, Xiao Huang, Gang Lu, Jian Yang, Xin Lu, Qihua Xiong, and Hua Zhang "Rapid and Reliable Thickness Identification of Two-Dimensional Nanosheets Using Optical Microscopy” ACS Nano 20137 (11), 10344-10353

[21] Li Y, Chernikov A, Zhang X, Rigosi A, Hill H M, van der Zande A M, Chenet D A, Shih E$\mathrm{M}$, Hone $\mathrm{J}$ and Heinz $\mathrm{T} F$ "Measurement of the optical dielectric function of monolayer transition-metal dichalcogenides: MoS2, MoSe2, WS2, and WSe2,” Phys. Rev. B 90, 2014.

[22] Schanda J “Colorimetry: understanding the CIE system,” John Wiley \& Sons, 2007. 
[23] Michael Stokes; Matthew Anderson; Srinivasan Chandrasekar; Ricardo Motta (November 5, 1996). "A Standard Default Color Space for the Internet - sRGB, Version 1.10" 